\title{
Compression of Frequency-Modulated Pulses using Helically Corrugated Waveguides and Its Potential for Generating Multigigawatt rf Radiation
}

\author{
S.V. Samsonov, ${ }^{1}$ A. D. R. Phelps, ${ }^{2}$ V. L. Bratman, ${ }^{1}$ G. Burt, ${ }^{2}$ G. G. Denisov, ${ }^{1}$ A.W. Cross, ${ }^{2}$ \\ K. Ronald, ${ }^{2}$ W. He, ${ }^{2}$ and H. Yin ${ }^{2}$ \\ ${ }^{1}$ Institute of Applied Physics, Russian Academy of Sciences, Nizhny Novgorod, 603950, Russia \\ ${ }^{2}$ Department of Physics, University of Strathclyde, Glasgow G4 ONG, Scotland, United Kingdom
}

(Received 13 July 2003; published 18 March 2004)

\begin{abstract}
A new method to generate ultrahigh-power microwave pulses compatible with mildly relativistic electron sources is proposed. This method involves a novel microwave compressor in the form of a metal helically corrugated waveguide, which can enhance the power of frequency-modulated nanosecond pulses up to the multigigawatt level. The results of the proof-of-principle experiments at kilowatt power levels are in good agreement with theory.
\end{abstract}

DOI: 10.1103/PhysRevLett.92.118301

Multigigawatt pulsed $\mathrm{rf}$ power, which has recently become available due to the development of relativistic electronics, opens up many new opportunities both for fundamental studies and future applications [1]. For example, it is important to recall that the power $P_{0}=$ $m^{2} c^{5} / e^{2}=8.7 \mathrm{GW}(m, e$ are electron mass and charge, respectively, and $c$ is speed of light) focused on an area of the order of the wavelength squared imparts a relativistic oscillatory velocity to electrons. Power at the multi-GW level was obtained at relatively long wavelengths of 3-10 $\mathrm{cm}$ when using electron beams with particle energies higher than $1 \mathrm{MeV}$ and currents of tens of $\mathrm{kA}$ that were available at a small number of unique high-current accelerators. In this Letter, an alternative method of producing multi-GW pulses is proposed. It uses the amplification of significantly lower power radiation, which is generated with a certain frequency modulation during the pulse. Compression of this pulse is achieved after propagation in a waveguide with the proper frequency dispersion. If a compression factor $K_{p}=$ $10-100$ is possible, then the input pulse power required will be of the order of hundreds of MW. Such pulses are produced routinely in Cherenkov relativistic traveling wave amplifiers (TWTs) or backward-wave oscillators (BWOs), driven with modest energy $0.5-1.0 \mathrm{MeV}$ electron beams [2,3]. However, compression of power from these devices requires additionally a sufficiently broadband frequency modulation within the pulse. A novel rf compressor in the form of a helically corrugated cylindrical waveguide can have the necessary dispersion while simultaneously it can sustain high strength rf fields as well as possessing a low level of reflections. Similar helically corrugated waveguides were intensively studied for gyro-TWTs [4].

Consider compression of a quasimonochromatic pulse with frequency, monotonously varying in time from $\omega_{1}$ to $\omega_{2}$. If the wave group velocity in the dispersive medium is an increasing function of frequency, $v_{\mathrm{gr}}\left(\omega_{2}\right)>$ $v_{\mathrm{gr}}\left(\omega_{1}\right)$, then the tail of the pulse will overtake its leading
PACS numbers: $84.40 . \mathrm{Ik}, 41.20 . \mathrm{Jb}, 41.60 . \mathrm{Cr}, 84.40 . \mathrm{Fe}$

edge, resulting in pulse shortening and a corresponding growth in amplitude if the losses are sufficiently low. To produce ultrahigh powers, the compressor characteristics should be optimized in order to achieve a maximum power compression factor at reasonable energy losses. Among the various methods of compression (see, e.g., [5]), the use of a metal waveguide is attractive because of its capability of handling high power, as well as its relative compactness and simplicity. In early studies, conventional regular waveguides were investigated as pulse compressors for radars [6]. In this case, the operating frequencies should be very close to the waveguide cutoff where the dispersion is sufficiently strong. It was also found that the optimum law of the frequency modulation, to ensure that the inverse group velocity, $\left(1 / v_{\text {gr }}\right)$, or delay time, is a linear function of time, was essentially nonlinear. Maximizing the time compression factor for a relatively long input pulse required a very large length, which led to a good signal to noise ratio but had high losses and, hence, a low energy efficiency.

The compression of a frequency-swept pulse can be approximated by the evolution of a plane wave $u(z, t)=$ $A(z, t) \exp (-i \omega t+i k z)$ in a linear dispersive medium, $k=k(\omega)$. When propagating through the medium, the wave can be represented in the form (see, e.g., [7])

$u(z, t)=\frac{1}{2 \pi} \int_{-\infty}^{+\infty} \int u_{0}\left(t^{\prime}\right) \exp \left[-i \omega\left(t-t^{\prime}\right)+i k(\omega) z\right] d t^{\prime} d \omega$,

where $u_{0}(t)=u(z=0, t)$ is the input signal. Preliminary estimations and optimization of the compressor length and the input pulse can be, however, performed with a simple kinematic approach, considering a rectangular input pulse, $u_{0}(t)=A_{0} \exp [-i \varphi(t)] t \in\left(0, \tau_{0}\right)$, as a train of "particles" having different velocities and entry times according to the dependence of the group velocity on time. The latter function is determined both by the dispersion of the medium, $v_{\mathrm{gr}}(\omega)=(d k / d \omega)^{-1}$, and the frequency modulation in the input pulse, $\omega(t)=d \varphi / d t$. 
Following this approach, the maximum pulse compression occurs when all the particles meet each other at the same distance. For this, the inverse group velocity of a particle should be a linear function of its entry time as follows:

$$
\frac{1}{v_{\mathrm{gr}}}=\frac{1}{v_{1}}-\left(\frac{1}{v_{1}}-\frac{1}{v_{2}}\right) \frac{t}{\tau_{0}} .
$$

The meeting point of particles is positioned at the distance $L=V \tau_{0}$, where $V=v_{1} v_{2} /\left(v_{2}-v_{1}\right)$. The corresponding "kinematic" pulse width at this point is equal to zero. In fact, the minimum achievable width is inversely proportional to the spectrum width of the input pulse and for sufficiently large frequency variation, $\Delta f \gg 1 / \tau_{0}$, it can be approximated as $\tau_{\min } \approx 1 / \Delta f$, where $\Delta f=\left|\omega_{2}-\omega_{1}\right| / 2 \pi$. For a lossless medium, the law of conservation of energy and the formula for $\tau_{\min }$ determine the maximum power compression

$$
K_{p}=\left|u_{\max }\right|^{2} / A_{0}^{2}=\Delta f \tau_{0} .
$$

Even for a limited modulation band, $\Delta f$, the power compression is unrestricted and goes up with an increase in the input pulse duration. In this case, however, compressor length $L$ also grows, and correspondingly the medium losses should be taken into account:

$$
K_{p}=\left|u_{\max }\right|^{2} / A_{0}^{2}=\Delta f \tau_{0} e^{-\bar{\gamma} L},
$$

where $\bar{\gamma}$ is the decrement averaged within the interval $\left[f_{1}, f_{2}\right]$. Substituting the pulse width as $\tau_{0}=L / V$, we derive the compression factor as a function of length, which has maximum

$$
K_{p \max }=\frac{\Delta f e^{-1}}{\bar{\gamma} V}
$$

at the length $L_{\mathrm{opt}}=1 / \bar{\gamma}$. In this case, the maximum energy efficiency is $e^{-1}$, or $37 \%$ (the rest of the input energy dissipates because of Ohmic losses). It should be noticed again that for pulse compression in smooth waveguides the operating frequencies are close to the cutoff, and the group velocity is usually less than $0.6 c$. In this range, the decrement of wave damping can be approximated by a linearly growing function of the inverse group velocity. At optimum modulation, when the inverse group velocity is a linear function of time (2), this approximation gives simply $\bar{\gamma}=\left(\gamma_{1}+\gamma_{2}\right) / 2$, where $\gamma_{1,2}$ are the decrements for the start and final frequency, respectively. Thus, for a chosen mode and optimum modulation, the maximum power compression can be estimated by an analytical function (5) of the frequencies, $\omega_{1}, \omega_{2}$, or the normalized group velocities, $v_{\text {gr1 }} / c, v_{\text {gr2 }} / c$ (Fig. 1). The simple analytical simulations agree very well with results of the nonapproximated 1D model, i.e., double integration of Eq. (1). For the $\mathrm{TE}_{1,1}$ mode of a copper $X$-band circular waveguide, a compression factor of nearly 100 can be achieved at a reasonable length of a

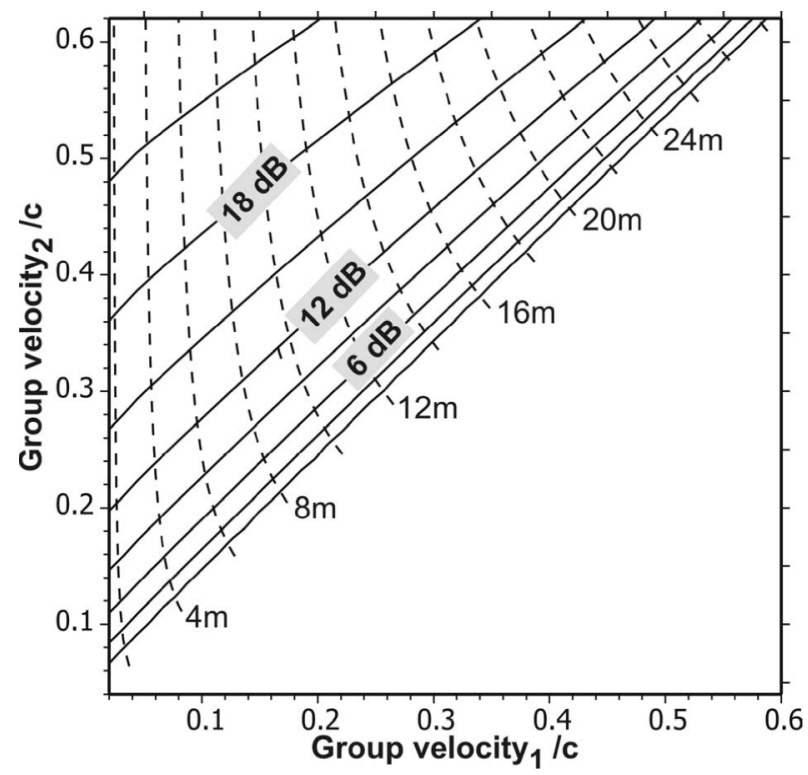

FIG. 1. Contours of maximum power compression ratio (solid lines) and optimum length (dashed lines) for a circular copper waveguide $19.2 \mathrm{~mm}$ in diameter.

few meters. For compression higher than 10, the output pulse duration amounts to $0.5-3$ ns. However, a serious drawback of a smooth waveguide as a powerful compressor is its operation very close to the cutoff. In optimum cases, the frequency at the beginning of an input pulse should be only $0.5 \%-1 \%$ above the cutoff frequency. If one uses such a compressor at the output of a powerful amplifier, then the low-frequency part of the amplification band will be reflected back to the amplifier resulting in its possible parasitic self-oscillation ( $\mathrm{rf}$ isolation using unidirectional elements is impossible at very high power). If a relativistic BWO is used as a source of frequencymodulated pulses for a smooth-waveguide compressor, then the necessary frequency sweep can be produced only by using a difficult-to-realize accelerating voltage modulation.

The two combined problems of wave reflection from a compressor and optimum frequency modulation can be solved using a waveguide with a special helical corrugation of its inner surface. Such a corrugation resonantly couples a pair of partial modes of a smooth waveguide resulting in a frequency region where the dispersion characteristic of one mode gradually transforms into that of the other mode [4] (Fig. 2). If the group velocity of these partial modes significantly differs from each other, then in the region of their coupling the group velocity of the eigenwave is a rapidly varying function of frequency, which is attractive for compressor realization. The helical symmetry allows the frequency regions with zero or negative group velocity of the eigenwave to be avoided, which ensures good rf matching of the compressor with the input source over a sufficiently wide frequency band. 

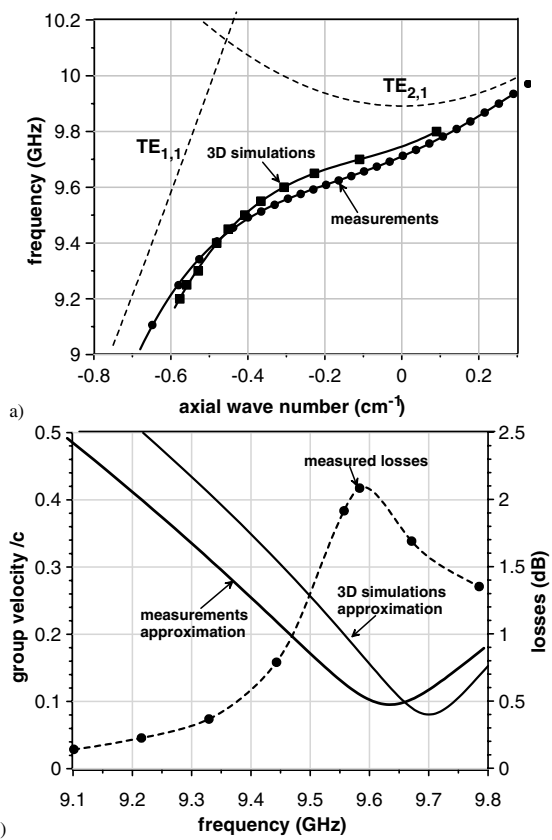

FIG. 2. (a) Dispersion diagram for the operating helically corrugated waveguide (b) group velocity derived from the curves in (a) and losses (in $\mathrm{dB}$ ) for a $2.08 \mathrm{~m}$ long waveguide as functions of frequency.

Operation of the compressors based both on a smooth and a helically corrugated waveguide was studied in an experiment. A conventional $X$-band TWT with an output power of about $1 \mathrm{~kW}$ driven by a solid-state frequencytunable oscillator was used for generating an input pulse for the compressors (Fig. 3). A pulse generator produced a sweep voltage, which changed almost linearly from $U_{1}$ to $U_{2}$. Both values as well as the transition time were smoothly controllable over a sufficiently wide range. This voltage was applied to a special pin of the solidstate rf source to control its oscillation frequency. An important feature of the rf oscillator was the limitation in the rate at which a smooth frequency sweep could be achieved and was found to be restricted to a value of

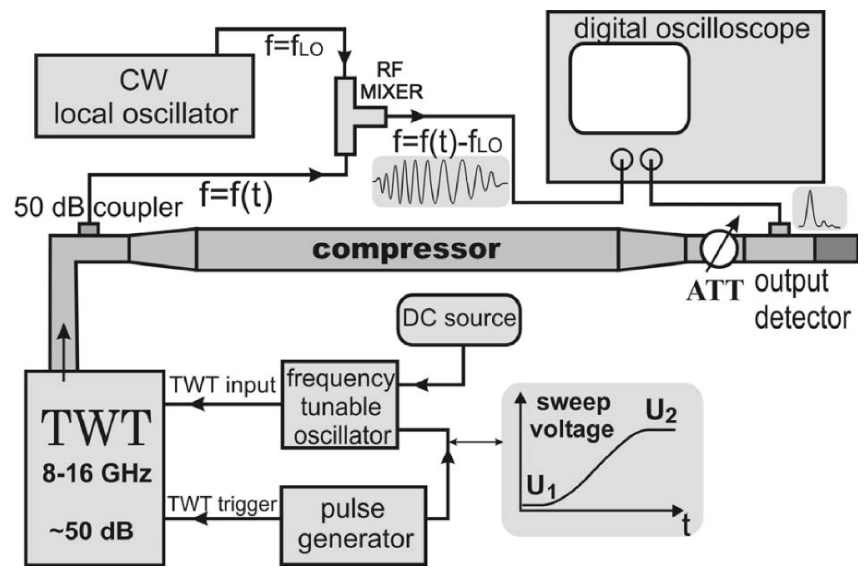

FIG. 3. Experimental setup.
7-9 MHz/ns. The solid-state source was unable to be switched on and off sufficiently quickly, and therefore a quasirectangular input pulse for the compressors was formed by triggering the TWT synchronized with the sweep voltage. The frequency modulation in the input pulse was monitored using a heterodyne technique (Fig. 3) and a fast digital oscilloscope. Power as a function of time for the input and output signals was digitally recovered from the detector traces using the detector calibration.

In the experiments, a circular aluminum waveguide $19.2 \mathrm{~mm}$ in diameter with the $\mathrm{TE}_{1,1}$ operating mode as well as a helically corrugated waveguide $29.4 \mathrm{~mm}$ in mean diameter were studied as pulse compressors. For the circular waveguide, the optimized compressed power varied within $\pm 15 \%$ for lengths of $4.95-9.90 \mathrm{~m}$ and had a maximum at a length of $6.60 \mathrm{~m}$. The compressed pulse of $2.5 \mathrm{~ns}$ width with a power gain of 7 contained about $35 \%$ of the input energy and was in very good agreement with the simulations (Fig. 4). The simulations included both the measured waveguide losses, corresponding to a wall conductivity of 0.35 of the conductivity of pure copper, and the measured frequency modulation of the optimized input pulse. Some transition region before the linear part of the sweep voltage pulse provided a frequency time dependence, which was close to the optimum law determined by Eq. (2). The achieved power compression was mainly limited by the available frequency sweep rate. For a copper waveguide and optimum frequency modulation
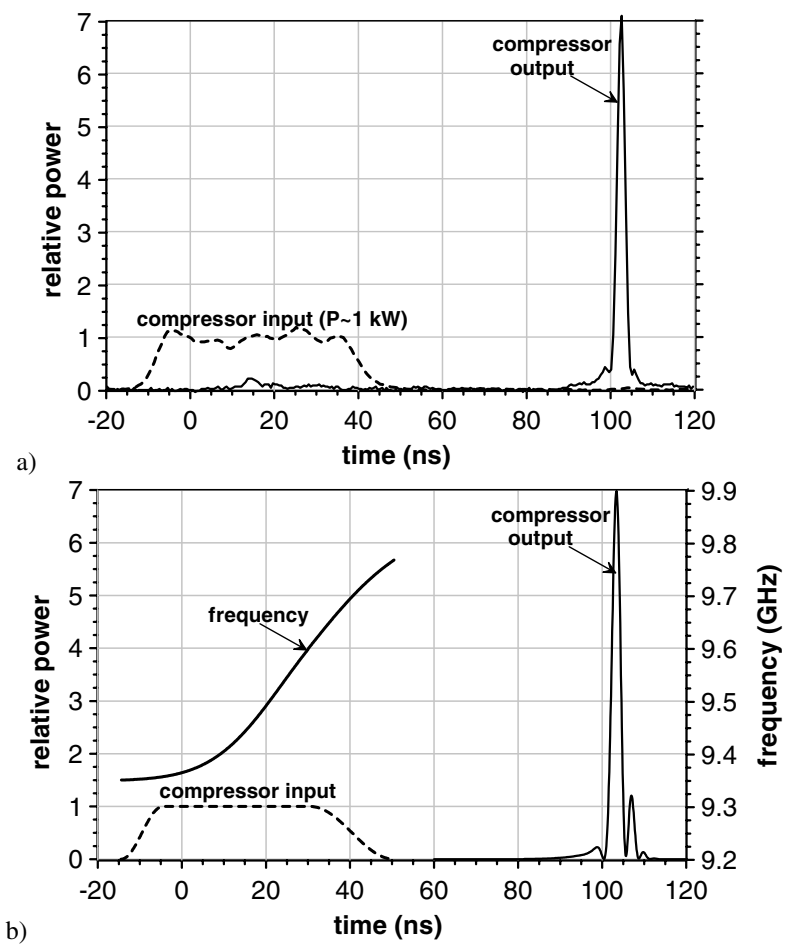

FIG. 4. Pulse compression in the circular waveguide: (a) experimental results; (b) simulation results. 


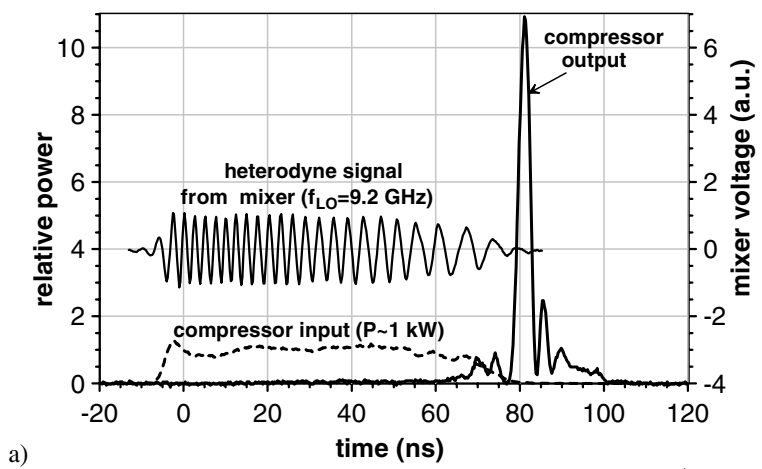

a)

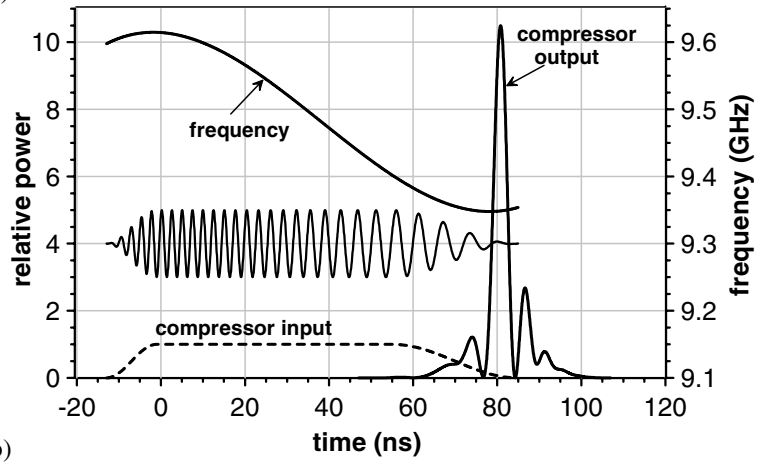

FIG. 5. Pulse compression in the helically corrugated waveguide: (a) experimental results; (b) simulation results.

with a sweep rate up to $20 \mathrm{MHz} / \mathrm{ns}$, the power gain would increase up to 40 .

For the helical compressor, a threefold sinusoidal corrugation coupled a circularly polarized $\mathrm{TE}_{1,1}$ mode having large group velocity with a near-cutoff counterrotating $\mathrm{TE}_{2,1}$ mode resulting in a frequency region of $5 \%-6 \%$ where the eigenwave group velocity changed from $0.1 c$ to $0.5 c$ (Fig. 2). The inner surface of the waveguide used was represented in cylindrical coordinates $r$, $\varphi, z$ as follows: $r(\varphi, z)=14.7+1.4 \sin (2 \pi z / 28.9-3 \varphi)$ (all dimensions are in millimeters). The copper helical waveguide had a total length of the regular corrugation of $2.08 \mathrm{~m}$ bounded from each side by four-period sections with linearly increasing/decreasing amplitude of the corrugation. The input radiation was launched from a WG16 waveguide and passed first through a rectangularto-circular adapter and then through a polarizer which converted the linearly polarized $\mathrm{TE}_{1,1}$ mode to a circularly polarized mode. At the output, the opposite transition took place. The most favorable region for pulse compression in helical waveguides is the part of the dispersion characteristic where the group velocity has a negative gradient as a function of frequency [Fig. 2(b)]. Correspondingly, the frequency modulation in the input pulse should have a negative slope in contrast to the case of a smooth waveguide. Experimental optimization at the fixed compressor length resulted in a compressed pulse of $3 \mathrm{~ns}$ duration with a peak power 10.9 times higher than the averaged power in the input pulse [Fig. 5(a)]. This pulse excluding the side lobes contained $44 \%$ of input energy. The measurements are in very good agreement with the simulations [Fig. 5(b)], where the frequency modulation was taken from analysis of the heterodyne signal and the operating wave dispersion including losses taken from "cold" microwave measurements. The simulations predict that with 2 times faster frequency sweep at the end of the pulse the power compression ratio and efficiency can be increased to 18.7 and $65 \%$, respectively.

For a helical compressor, the optimum negative frequency sweep can quite naturally be realized at the falling edge of a relativistic BWO pulse. For example, if a BWO generates hundreds of MW during 50 ns over a voltage drop from 700 to $400 \mathrm{kV}$, this frequency-modulated radiation can be compressed up to 10-20 times in power with efficiency higher than 50\%. In addition, because of its reflectionless properties, a helical compressor can be used effectively at the output of a powerful amplifier. A favorable wave dispersion can be also synthesized for higher-order modes resulting in an increase of the helical waveguide diameter by 1.5-2 times (without significant overlapping of the coupling bands) and correspondingly in an enhancement of its rf breakdown strength. A combination of a moderately relativistic BWO or TWT with a helical waveguide compressor may result in a multigigawatt short-pulse microwave source which is attractive for a number of applications including radars and plasma chemistry [1].

This work was supported by a grant of the Presidium of Russian Academy of Sciences, the University of Strathclyde and EPSRC, U.K.

[1] Applications of High-Power Microwaves, edited by A.V. Gaponov-Grekhov and V. L. Granatstein (Artech House, Boston, 1994).

[2] C. Chen, G. Liu, W. Huang, Z. Song, J. Fan, and H. Wang, IEEE Trans. Plasma Sci. 30, 1108 (2002).

[3] E. B. Abubakirov, A. N. Denisenko, M. I. Fuks, N. G. Kolganov, N. F. Kovalev, M. I. Petelin, A.V. Savelyev, E. Shamiloglu, E. I. Soluyanov, and V.V. Yastrebov, IEEE Trans. Plasma Sci. 30, 1041 (2002).

[4] V. L. Bratman, A. W. Cross, G. G. Denisov, W. He, A. D. R. Phelps, K. Ronald, S. V. Samsonov, C. G. Whyte, and A. R. Young, Phys. Rev. Lett. 84, 2746 (2000).

[5] M. K. Thumm and W. Kasparek, IEEE Trans. Plasma Sci. 30, 755 (2002).

[6] R. A. Bromley and B.E. Callan, Proc. IEE 114, 1213 (1967).

[7] E. Hecht, Optics (Pearson Addison Wesley, Reading, MA, 2004), 4th ed. 